\title{
An Extended Tropospheric Scintillation Model for Free Space Optical Communication Systems
}

\author{
John O Famoriji ${ }^{1}$, Yekeen O Olasoji ${ }^{2}$, Michael O Kolawole ${ }^{3}$ \\ ${ }^{1}$ Department of Electrical and Electronics Engineering, Afe Babalola University, P.M.B. 5454, Ado, Ekiti \\ State, Nigeria. \\ ${ }^{2,3}$ Department of Electrical and Electronics Engineering, Federal University of Technology \\ P. M. B. 704, Akure, Ondo State, Nigeria. \\ *Author Corresponding, Email: famorijijohn@yahoo.co.uk ${ }^{1}$, yoolasoji@futa.edu.ng ${ }^{2}$ \\ yoolasoji@yahoo.com², mkolawole@futa.edu.ng ${ }^{3}$,kolawolm@yahoo.com³
}

\begin{abstract}
Fluctuations caused mostly by tropospheric scintillation at the free space optical receiver end have been a major problem in the rapid development of telecommunication and the increasing demands for larger bandwidth is forcing the use of free space optical (FSO) technology. This paper examined existing tropospheric scintillation models of Karasawa, Van de Kamp model, Otung, Ortgies and ITU-R, and discovered that all of them operate at the microwave range, which limits their application in FSO laser beam technology that operates in PHz frequency-range. ITU-R model was later selected owing to its global application and modified for use in FSO communication system. The new model can serve as basis for communication engineers to use as platform in the link budgetary for planning and design of low margin systems of free space optical communication link.
\end{abstract}

Keywords: Signal fluctuation, Tropospheric Scintillation Model, FSO, ITU-R

\section{Introduction}

The uptake of free-space optical (FSO) transmission is constrained by attenuation due to fog and cloud as well as scintillation fading. Scintillation is the random optical-power fluctuations in signal amplitude, which leads to image fluctuations at the FSO receiver end caused by atmospheric turbulence as a result of refractive index variation [1]. In the assessment of a link budget, it is of great importance to quantify the losses caused by scintillation in terms of power loss. This is to account for additional power that will be needed to overcome scintillation effects and thus to reach the required performance. Scintillation loss is a major issue to address in optical wireless communication system.

Scintillation is categorized into two types: ionospheric scintillation, and tropospheric scintillation. Ionospheric scintillation is a rapid fluctuation of radio-frequency signal amplitude and/or phase, generated as a signal traverses through the ionosphere causing small-scale irregularities in electron density. The presence of these charged particles makes the ionosphere an electrical conductor, which supports electric currents and affects radio waves [2], thereby affecting trans-ionospheric radio signals of frequencies up to $6 \mathrm{GHz}$. Tropospheric scintillation is a rapid fluctuation of signal amplitude and/or phase due to turbulent irregularities in temperature, humidity, and pressure, which translate into small-scale variations in refractive index [3]. In the tropics where this research study is conducted, as well as in the equatorial regions, the humidity fluctuations are important because they cause random degradation and enhancement in signal amplitude and phase received on a satellite-earth link, as well as degradation in performance of large antennas. Moreover, on the line of sight linkup through 10 $\mathrm{GHz}$ and on earth-space paths at frequencies above $50 \mathrm{GHz}$, the tropospheric scintillation is often detected [4].

This research work focuses on the tropospheric scintillation; as such, what constitutes a troposphere is described as follows. The troposphere is the lowest and unstable layer of Earth's atmosphere, where most of the weather phenomena, systems, convection, turbulence and clouds occur. The troposphere contains $99 \%$ of the water vapour-whose concentrations vary with latitudinal position-in the atmosphere [2]. The height of the troposphere varies with location being higher over warmer areas and lower over colder areas; it ranges between 10km-12km [5]. 
Tropospheric scintillation occurs with or without rain (clear air or sky); it is therefore a serious concern in free space optical channel which impairs the availability and reliability of the system. However, clear air turbulence has long been identified as a primary source of scintillation. Models that focus on clear air effects, as well as fair-weather cumulus clouds crossing the transmission link, include those of [3], [6], [7], [8]. Research studies continue, though, in predicting tropospheric scintillation both theoretical and empirical. Regardless of the methods employed, inclusion of the main link parameters (for example, the frequency, elevation angle and antenna diameter) and meteorological data (for instance, the humidity at ground level and mean temperature) are needed in order to obtain reliable scintillation prediction. Tropospheric scintillation is therefore a signal propagation impediment, which must be accounted for in order to complete the link budget for design of low margin systems.

\section{Tropospheric Scintillation Models}

There are many prediction models that have been proposed over the years in order to estimate the statistical distributions of scintillation. The required input parameters needed for these models are signal frequency $f(\mathrm{GHz})$, antenna diameter $\mathrm{D}(\mathrm{m})$, path elevation angle $\Theta$ $(\mathrm{deg})$, average temperature $\left({ }^{\circ} \mathrm{C}\right)$, and average relative humidity $(\%)$ which are readily available. However, all the proposed tropospheric scintillation models are usable and applicable for signals frequencies in the $\mathrm{GHz}$ range because of the higher wavelength (microwave) as compared to low wavelength in which FSO system operates. The losses are more pronounced on FSO communication systems, which should be accounted for. Some of the current tropospheric scintillation models are presented in the following subsections.

\subsection{Karasawa Scintillation Prediction Model}

This is a measurement based prediction model made in the year 1983, Yamaguchi city of Japan at an elevation angle of $6.5^{\circ}$, frequencies of 11.5 and $14.23 \mathrm{GHz}$ and an antenna diameter of $7.6 \mathrm{~m} \mathrm{[1]}$. The following prediction formulae were derived using data:

$$
\sigma_{\text {pre }}=0.0228\left(0.15+5.2 \times 10^{-3} N_{\text {wet }}\right) d B f^{0.45} \sqrt{G\left(G_{c}\right)} / \sin ^{1.3} \varepsilon
$$

where:

$\sigma_{\text {pre }}=$ The predicted signal standard deviation or scintillation intensity

$f=$ Frequency $(\mathrm{GHz})$

$\varepsilon=$ Apparent elevation angle (degree)

$G\left(G_{c}\right)=$ Antenna averaging

$G_{c}=$ Effective antenna diameter given by:

$$
D_{c}=D \sqrt{\eta}
$$

$D=$ Geometrical antenna diameter $(\mathrm{m})$

$\eta=$ Antenna aperture efficiency

This prediction model indicated that the antenna averaging function also depends on the elevation angle and the height of the turbulence to be $2000 \mathrm{~m}$.

If $\varepsilon<5^{0}, \sin \varepsilon$ in Eqn. (1) should be replaced by:

$$
\sin \varepsilon+\sqrt{\left(\sin ^{2} \varepsilon+\frac{2 h}{R_{e}}\right)} / 2
$$

where,

$h=$ Height of the turbulence $(\mathrm{m})$

$R_{e}=$ Effective earth radius $=8.5 \times 10^{6} \mathrm{~m} \mathrm{[6]}$

The effective earth radius varies with latitude, i.e. as one move away from the equator. Nigeria location is above the equation with $R_{e}$ of $6378 \mathrm{~km}$, which is fractionally different from that of Japan which Karasawa quoted.

The following equation is the wet term of the refractivity at ground level: 
$N_{\text {wet }}=\frac{22790 U e^{(19.7 t / t+273)}}{(t+273)^{2}}(p p m)$

where

$N_{\text {wet }}=$ Relative humidity (\%) due to water vapor in the atmosphere

$t=$ Temperature $\left({ }^{\circ} \mathrm{C}\right)$

$\mathrm{U}=$ Relative humidity (\%)

Karasawa et al (1988) also presented that the meteorological input parameters should be averaged over a period in the order of a month so the model does not predict short-term scintillation variations with daily weather changes. The equations for the scintillation enhancement $\left(n\left(p^{+}\right)\right)$and scintillation fading $\left(n\left(p^{-}\right)\right)$are respectively expressed as follows:

$$
\begin{aligned}
& n\left(p^{+}\right)=-0.0597(\log (100-p))^{3}-0.0835(\log (100-p))^{2} \\
& -1.258(\log (100-p))+2.672, \text { for } 50<p \leq 99.99 \\
& n\left(p^{-}\right)=-0.061(\log p)^{3}+0.072(\log p)^{2}-1.71(\log p)+3.0, \text { for } 0.01<p \leq 50
\end{aligned}
$$

To determine the cumulative time distribution for the scintillation enhancement $(X(p))$ and scintillation fade $\sigma_{\text {pre }}$ has to be included in Eqns. (5) and (6); specifically,

$$
\begin{aligned}
& X(p)=n\left(p^{+}\right) \times \sigma_{\text {pre }} \\
& X(p)=n\left(p^{-}\right) \times \sigma_{\text {pre }}
\end{aligned}
$$

Generally, it could be observed that the model approach was on Intelsat and applicable to wide regions under different climate most especially where the research was carried out. However, the data used here are for four seasons (namely: Winter, Autumn, Summer and Spring) only and does not include desert or tropical region.

\subsection{Ortgies Scintillation Prediction Models}

Ortgies (1993) presented two models: Ortgie-Refractivity (Ortgie-R) and OrtgieTemperature (Ortgie-T). The experiment was conducted on Olympus satellite measurements at Darmstadt, Germany. The frequencies used were 12.5, 20 and $30 \mathrm{GHz}$. Ortgies applied a lognormal probability density function (pdf) for long term distribution of scintillation intensity parameters; $\mu$ and $s$ which are mean and standard deviation of $\ln \left(\sigma_{x}^{2}\right)$ respectively [9]. The two models are based on direct proportional relationships that exist between mean surface measurement and monthly mean normalized log variance of scintillation. Ortgies-T model takes the monthly mean surface temperature $(\mathrm{T})$ as a predictor:

$$
\ln \left(\sigma_{\text {pre }}^{2}\right)=\ln \left[g^{2}(x) k^{1.21}(\sin \theta)^{-2.4}\right] 12.5+0.0865(T)
$$

Whereas the Ortgies-N model uses monthly mean log-variance of signal log-amplitude to monthly mean wet component of surface refractivity $\left(N_{\text {wet }}\right)$ as a predictor:

$$
\ln \left(\sigma_{\text {pre }}^{2}\right)=\ln \left[g^{2}(x) k^{1.21}(\sin \theta)^{-2.4}\right]-13.45+0.0462\left(N_{\text {wet }}\right)
$$

However, the models are not appropriate for tropical or desert climate, though it includes meteorological parameters, $N_{\text {wet }}$.

\subsection{Otung Scintillation Prediction Model}

Otung [10] worked on the prediction of tropospheric amplitude scintillation. A simple expression was proposed for the annual and worst-month cumulative distributions of scintillation fades $x_{-}$and enhancements $x_{+}$which are applicable to predict scintillation on a satellite link. The scintillation data were obtained at Sparshot, UK $(51.5850 \mathrm{~N}, 1.5033 \mathrm{~W})$ for a period of one year by the use of Olympus satellite $19.7704 \mathrm{GHz}$ beacon observed at elevation angle $28.74^{0}$. This model is related to the ITU-R model except a little modification in the elevation angle of the scintillation fade, expressed as: 


$$
\sigma_{\text {pre }}=\frac{\sigma_{\text {pre }} f^{\frac{7}{12}} g(x)}{(\sin \theta)^{11 / 12}}
$$

For annual distribution, the scintillation fades, $x_{-a}$ and scintillation enhancement, $x_{+a}$ are written as:

$$
\begin{aligned}
& x_{-a}=3.6191 \sigma_{\text {pre }} \exp \left(\frac{-9.50142 \times 10^{-4}}{p}[0.40454+0.00285 p] \ln (p)\right) \\
& \text { for } 0.01 \leq p \geq 50 \% \\
& x_{+a}=3.1782 \sigma_{\text {pre }} \exp (0.0359654 p-[0.272113-0.00438] \ln (p) \\
& \text { for } 0.01 \leq p \geq 50 \%
\end{aligned}
$$

where $a$ is the annual distribution, and $p$ is time percentage factor. For worst-month distribution, the scintillation enhancement and scintillation fade $\mathrm{x}_{+\mathrm{w}}$ and $\mathrm{x}_{-\mathrm{w}}$ respectively, are written as:

$$
\begin{aligned}
& x_{-w}=6.8224 \sigma_{\text {pre }} \exp \left(-10^{-4}\left[\frac{9.1312}{p}+1.8264 p^{2}\right]-\left[\frac{0.023027}{p}+0.51664\right] \ln (p)\right. \\
& \text { for } 0.003 \leq p \geq 50 \% \\
& x_{+w}=5.5499 \sigma_{\text {pre }} \exp \left(-10^{-4}\left[946.849 p+4.4974 p^{2}\right]+[0.02357 p-0.261135] \ln (p)\right. \\
& \text { for } 0.01 \leq p \geq 50 \%
\end{aligned}
$$

Whilst the Otung (1996) model provides worst-month and annual distributions of scintillation, it is not applicable to tropical climate condition.

\subsection{Van De Kamp Tropospheric Scintillation Model}

Van de Kamp et al. [3] deployed the ITU-R model in their prediction model but a small change in the elevation angle as in Eqn. (16). This model was derived and tested in four sites in different climates: Japan, United Kingdom, Finland, and Texas by scintillation measurements. Van de Kamp et al. [3] model introduced the cloud type information based on edited synoptic cloud reports, which observed that there was a scintillation correlation between the occurrence of scintillation and the presence of cumulus clouds. Also, Mayer [11] published an improved version of the Van de Kamp et al [3] model, and that heavy clouds are clouds with integrated water content larger than $0.7 \mathrm{~kg} / \mathrm{m}^{2}$. He incorporated $W_{h c}$ into the model, thus:

$$
\begin{aligned}
& \sigma_{p}=\frac{f^{0.45} \sqrt{g^{2}(D e)}}{\sin ^{1.3}(\varepsilon)} 0.98 \times 10^{-4}\left(N_{w e t}+Q\right) \\
& Q=-39.2+\left\langle W_{h c}\right\rangle Q
\end{aligned}
$$

where

$W_{h c}=$ Average water content of heavy clouds $\left[\mathrm{kg} / \mathrm{m}^{2}\right]$

$\langle x\rangle=$ Long-term (at least) average of the parameter $\mathrm{x}$

$Q=$ Long-term average parameter and hence constant for each site, so that all seasonal dependence of $\sigma_{p}$ is still represented by $N_{\text {wet }}$

Van de Kamp et al (1999) also adopted formulae for scintillation enhancement and scintillation fade depth. Specifically,

$$
\begin{aligned}
& a_{1}(p)=-0.0515\left(\log _{10} p\right)^{3}+0.206\left(\log _{10} p\right)^{2}-1.5-81 \log _{10} p+2.18 \\
& a_{2}(p)=-0.172\left(\log _{10} p\right)^{2}-0.454 \log _{10} p+0.274
\end{aligned}
$$

where

$a_{1}(p)$ and $a_{2}(p)$ are time percentage factors:

$E_{p}(p)=a_{1}(p) \sigma_{p}-a_{2}(p) \sigma^{2}{ }_{x}$ for $0.001 \leq p \leq 20$ 


$$
a_{2}(p)=a_{1}(p) \sigma_{p}+a_{2}(p) \sigma_{x}^{2} \text { for } 0.001 \leq p \leq 20
$$

where $E_{p}(p)$ and $a_{2}(p)$ are scintillation enhancement and scintillation fade depth, respectively. It was observed that the scintillation enhancement and scintillation fade depth in Van de Kamp et al [3] model are meant for the percentage factors from 0.001 till 20, but this is in contrast to Karasawa et al [6], Otung [10] and ITU-R [7] models whose percentage factor is between 0.001 and 50. Van de Kamp scintillation prediction model includes cloud information and has significant improvement on the accuracy of scintillation variance. However, the model is on experimental data from limited sites, may be as a result of scarcity of experimental data and cannot be used for tropical climatic condition.

\subsection{ITU-R Tropospheric Scintillation Model}

A tropospheric scintillation model was developed by international telecommunication union of radio section (ITU-R), which has frequencies between $7-14 \mathrm{GHz}$ and theoretical frequency dependence and aperture averaging effects, estimates the average scintillation intensity $\sigma_{\text {per }}$ over a minimum period of one month [1]. The input parameters required for this model are: signal frequency $f(\mathrm{GHz})$, antenna diameter $\mathrm{D}(\mathrm{m})$, path elevation angle $\theta$, average temperature $\left({ }^{0} \mathrm{C}\right)$ and average relative humidity $\mathrm{U}(\%)$ which are readily available. The elevations angle used for the model is between $4^{0}$ and $32^{\circ}$ and the antenna diameters used is between 3 and $36 \mathrm{~m}$. Also in the ITU-R scintillation model, the long term scintillation variance is expressed as a relationship with $N_{\text {wet }}$; which is a function of relative humidity $\mathrm{U}(\%)$ and temperature $\mathrm{t}\left({ }^{0} \mathrm{C}\right)$, measured at ground level (P. 618-10 2009):

$$
N_{w e t}=3.732 \times 10^{5} \frac{e}{T^{2}}
$$

For the temperature range of -20 to $50^{\circ} \mathrm{C}$, the ITU-R P453-9 defined the water vapour pressure as:

$$
e=0.01 \times U \times\left(6.1121 \exp \left[\frac{17.502}{t+240.97}\right]\right)
$$

where

$e$ : water vapour pressure $(\mathrm{hPa})$

$T$ : absolute temperature $(\mathrm{K})$

t: Celsius temperature $\left({ }^{0} \mathrm{C}\right)$

$\mathrm{U}$ : relative humidity (\%)

The standard deviation of the signal fluctuation due to scintillation is given by:

$$
\sigma=\sigma_{r e f} f^{\frac{7}{12}}\left[\frac{g(x)}{(\sin \theta)^{1.2}}\right](d B)
$$

where

$\sigma_{\text {ref }}=$ Normalized or reference standard deviation given by:

$$
\sigma_{\text {ref }}=3.6 \times 10^{-3}+N_{\text {wet }}(d B)
$$

$g(\mathrm{x})=$ Antenna averaging factor

$$
g(x)=\sqrt{3.86\left(x^{2}+1\right)^{\frac{11}{12}} \sin \left(\frac{11}{6} \arctan \frac{1}{x}\right)-7.80 x^{\frac{5}{6}}}
$$

where

$$
x=1.22 D_{\text {eff }}^{2}\left(\frac{f}{L}\right)
$$


Effective diameter

$$
D_{e f f}=\sqrt{\Pi} D
$$

$D$ is antenna aperture diameter; $\eta$ is antenna efficiency $(0 \leq \eta \leq 1)$.

$$
L=\frac{2 h_{L}}{\sqrt{(\sin \theta)^{2}+2.35 \times 10^{-4}}+\sin \theta}
$$

where;

$h_{L}=$ Height of the turbulent layer; $h_{L}=1000 \mathrm{~m}$

$\theta=$ Elevation angle

This model is applicable to wide regions of different climates. However, it cannot be used in tropical region, as well as in an atmosphere that is dry. It has an advantage of been used globally (i.e. it is applicable everywhere). Other tropospheric scintillation models (most especially those discussed above) are modification of ITU-R tropospheric scintillation model. However, all the proposed tropospheric scintillation models, including ITU-R model are usable and applicable for signals frequencies in the $\mathrm{GHz}$ range. So, because of the higher wavelength microwave possesses as compared to low wavelength in which FSO system operates, this may introduce higher absorption due to rainfall, scattering, reflection, refraction and fading, which in turn increases the unavailability and unreliability of the free space optical communication system. This rendered these models less applicable to FSO technology. Therefore, there is a need to propose a scintillation model that will fit in for FSO systems (Laser beam) which operates in $\mathrm{PHz}$ frequency range. This FSO scintillation model will account for the higher fluctuation of the amplitude and phase of the beam signal at the receiver end, so that wireless communication engineers can have a better platform to work with in order to reach their target, which is optimal performance by providing a reliable network and high quality of service.

\section{Research Method}

ITU-R tropospheric scintillation model-as stated in [1], i.e. Eqn. (22)-was used as a slave model to determine a scintillation model that fit-in for FSO spectrum. The model in its original form has its application in microwave ( $\mathrm{GHz}$ frequency range) but FSO laser or beam signal operates in $\mathrm{PHz}(0.1$ to $10 \mathrm{PHz})$ frequency range. The two cases are considered: ITU-R model with microwave mean frequency; and ITU-R model with FSO (laser beam) mean frequency range. The two results were added and averaged to determine the suitable tropospheric scintillation model for FSO communication systems.

All the parameters are as defined in Sec. 2.2.5. The efficiency is assumed to be unity though this may not be so in practice. Turbulent height $\left(h_{L}\right)$ was taken to be $1000 \mathrm{~m}$ as proposed by ITU-R. Elevation angle $\Theta$ under consideration is $30^{\circ}$, which is within the range of ITU-R. The temperature $\mathrm{t}$ and relative humidity $\mathrm{H}$ were set at $37.1^{\circ} \mathrm{C}$ and $24 \%$ respectively and antenna aperture diameter was taken to be $15 \mathrm{~m}$. Matlab Simulink Software Package was used for the simulation of the model under the two cases and plot of the standard deviation $\sigma$ against frequency $f$ for both microwave mean and laser mean frequencies were generated, also the average of the two cases was determined using the same Matlab software package.

\section{Results and Discussion}

Equation (24) is the slave model considered for the following two cases:

Case 1: Standard deviation $\sigma$ at microwave mean frequency spectrum (12 to $20 \mathrm{GHz}$ ) is denoted as $\sigma_{1}$, i.e.

$$
\sigma_{1}=\sigma_{r e f} f_{1}^{\frac{7}{12}}\left[\frac{g_{1}(x)}{(\sin \theta)^{1.2}}\right](d B)
$$

The simulation result is presented in Figure 1. 
Case 2: Standard deviation $\sigma$ at laser mean frequency spectrum $(0.1$ to $10 \mathrm{PHz})$ is denoted as $\sigma_{2}$.

$$
\sigma_{2}=\sigma_{r e f} f_{2}^{\frac{7}{12}}\left[\frac{g_{2}(x)}{(\sin \theta)^{1.2}}\right](d B)
$$

The simulation result is presented in Figure 2.

The two cases were averaged to give: $\frac{\sigma_{1}+\sigma_{2}}{2}$ (i.e. averaging Eqns. (29) and (30)):

$$
\frac{\sigma_{r e f}}{2(\sin \theta)^{1.2}}\left[f_{1}^{\frac{7}{12}} g_{1}(x)+f_{2}^{\frac{7}{12}} g_{2}(x)\right]
$$

It is justifiable to say, that since $f_{1}<<f_{2}$ and $g_{1}(x)<<g_{2}(x)$ then, it is ascertained to say that $f_{1}^{\frac{7}{12}} g_{1}(x)<<f_{2}^{\frac{7}{12}} g_{2}(x)$, and consequentially, if the above holds, we formulate a new tropospheric scintillation model suitable for free space optical communication system as:

$$
\sigma=\frac{\sigma_{r e f}}{2(\sin \theta)^{1.2}}\left[f^{\frac{7}{12}} g(x)\right]
$$

All the parameters are as defined by ITU-R except the frequency range which is now 1 to $5.5 \mathrm{PHz}$ as evident in Figure 3.

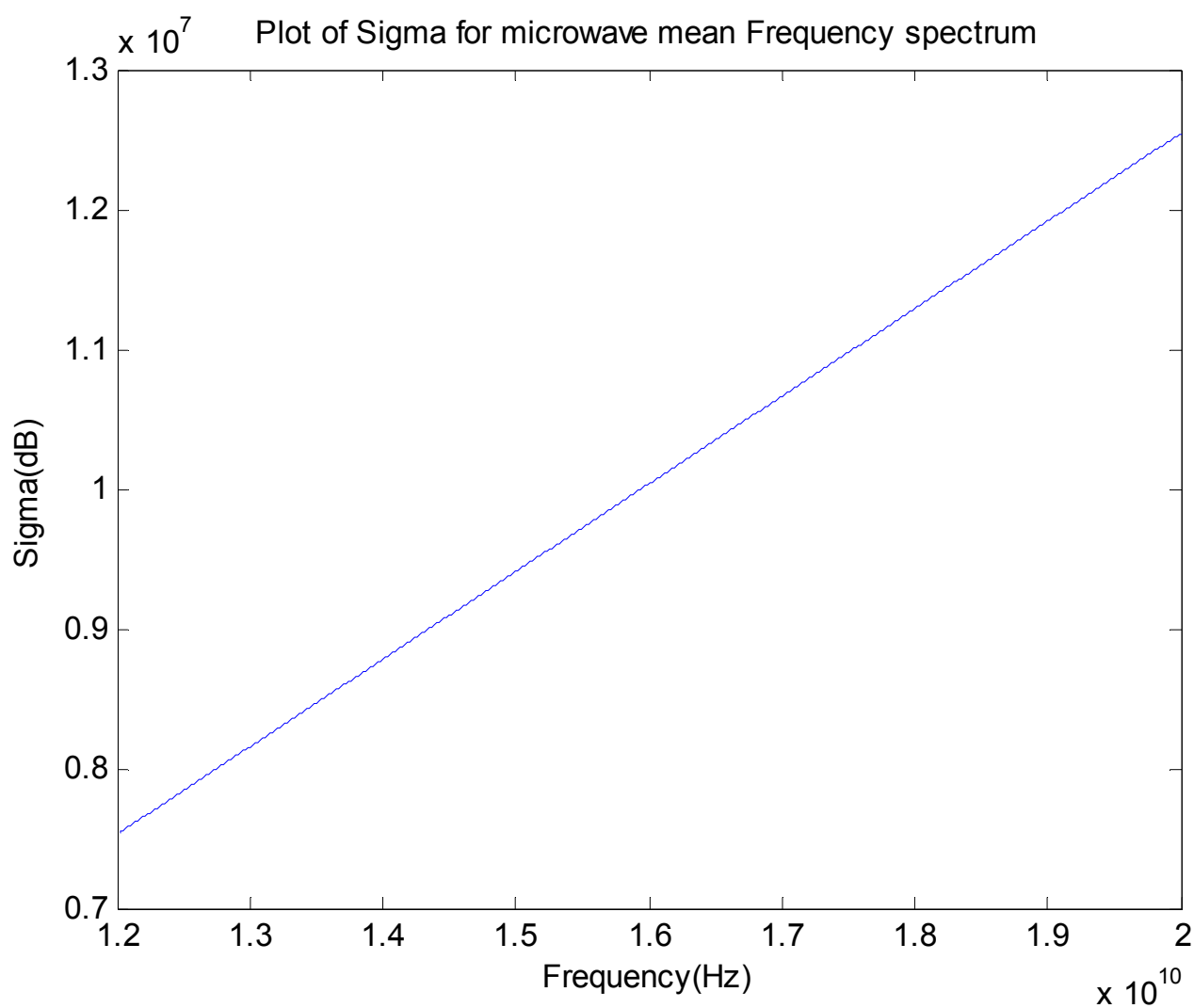

Figure 1. Plot of Sigma against Microwave Mean Frequency Spectrum 


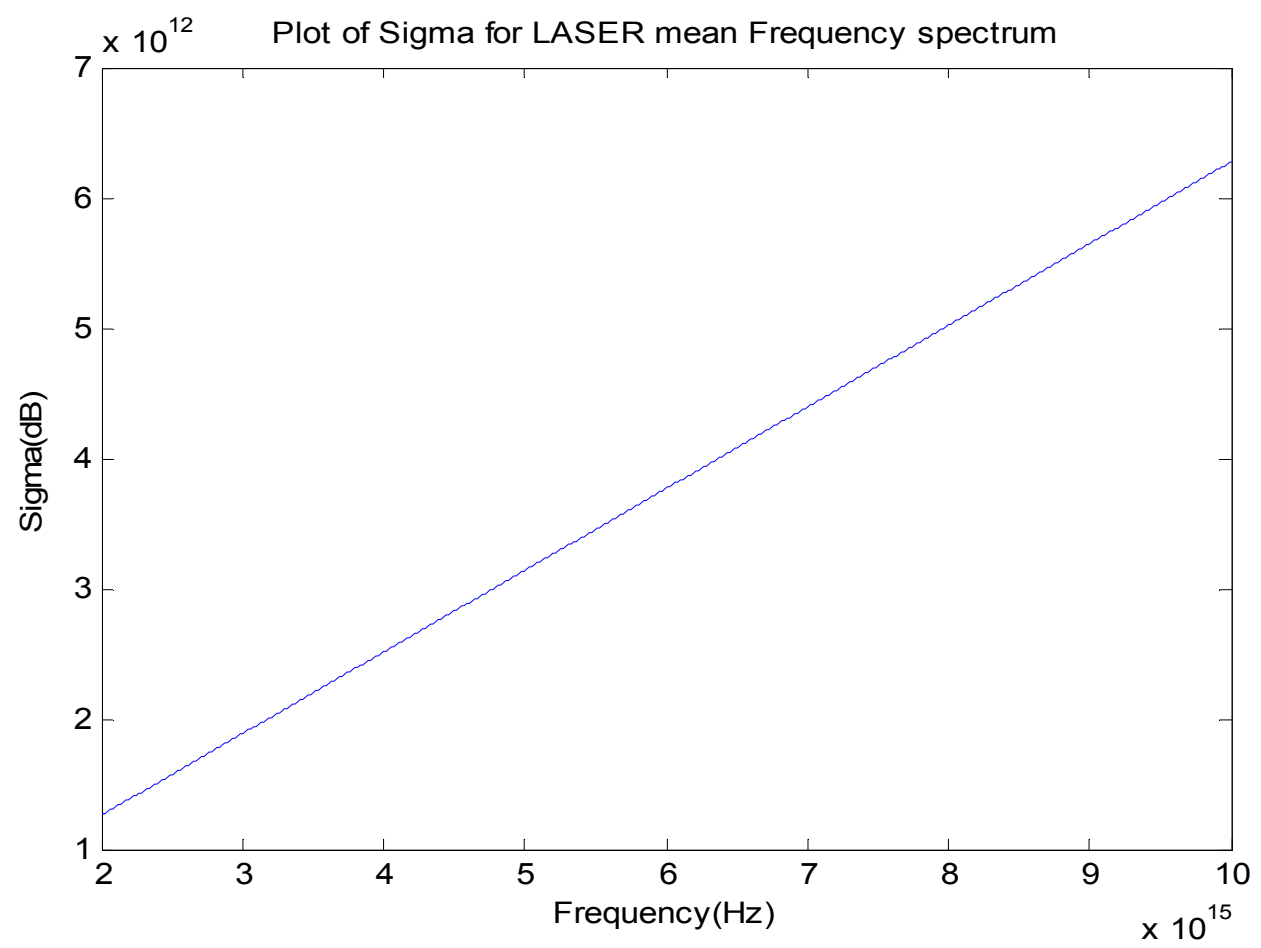

Figure 2. Plot of Sigma against LASER Mean Frequency Spectrum

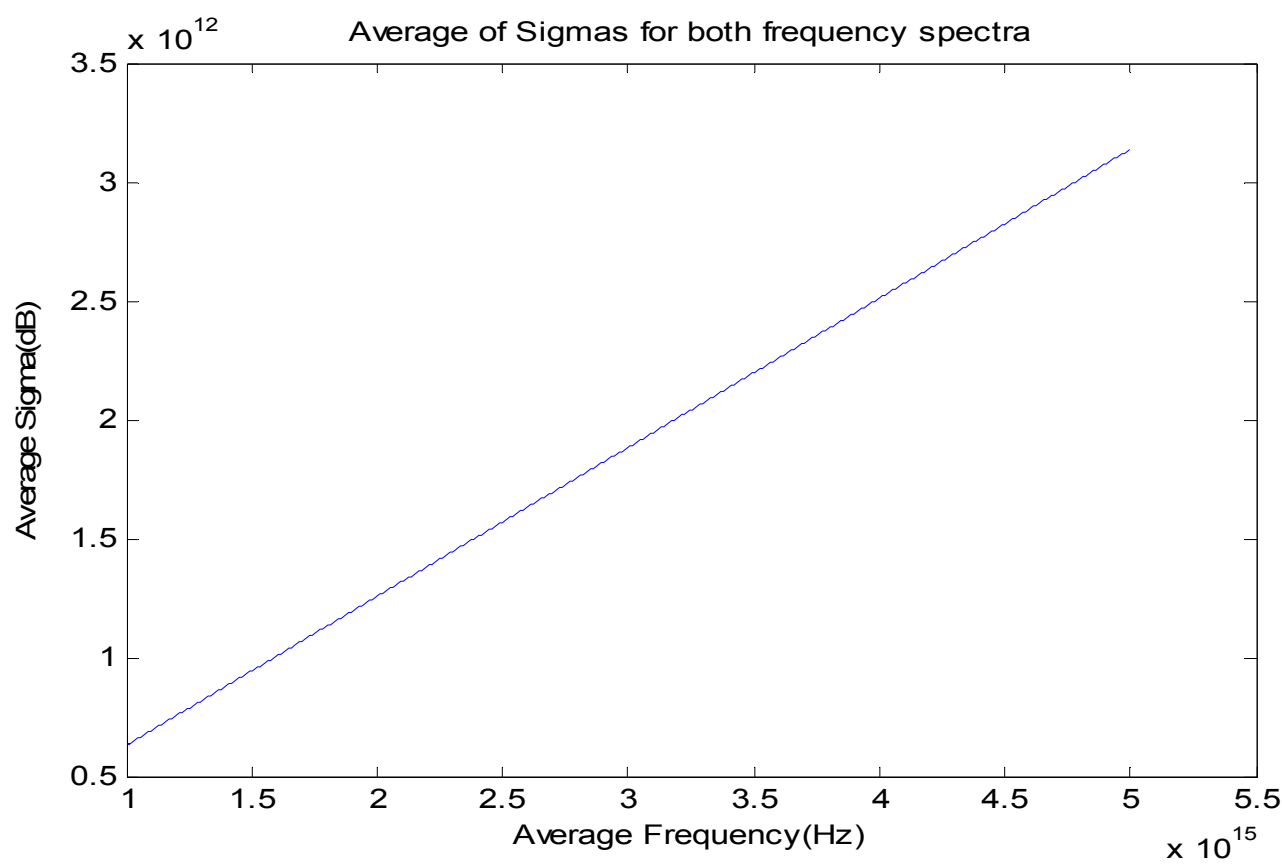

Figure 3. Average of Figure 1 and Figure 2

\section{Conclusion}

The uptake of free-space optical (FSO) transmission is constrained, among other things, by scintillation fading. Tropospheric scintillation is predominated in the tropics, which this paper investigated. This paper has presented a modified ITU-R tropospheric scintillation model 
that is useable at laser beam frequency spectrum (free space optical communication system). It provides basis for communication engineers to use as platform in the link budgetary for planning and design of low margin systems of free space optical communication link.

\section{References}

[1] Nadirah BAR, Rafiqul I, Mandeep JS and Hassan D. "Com.arison of Tropospheric Scintillation Models on Earth-Space Paths in Tropical Region". Research Journal of Applied Sciences, Engineering and Technology. 2012; 4(11): 1616-1623.

[2] Kolawole MO. "Radar systems, peak detection and tracking", Oxford: Elsevier Science. 2003.

[3] Van de Kamp MM, Tervonen JK, Salonen ET Pirate Baptista JPV. "Improved Models for Long-term Prediction of Tropospheric Scintillation on Slant Paths. IEEE Transaction on Antenna Propagation. 1999; 47(2): 249-260.

[4] Jr LJI. "Satellite Communication Systems Engineering: Atmospheric Effects, Satellite Link Design and System Performance". John Wiley and Son Ltd Publications, Washington DC, USA. 2008.

[5] Wallace JM and Hobbs PV. "Atmospheric Science: An Introduction Survey. $2^{\text {nd }}$ Edition, New York. (2006),

[6] Karasawa Y, Yamada M and Allnut JF. "A new Prediction method for Tropospheric Scintillation on Earth-Space Paths. IEEE Antenna propagation. 1988; 36(11): 1608-1614.

[7] ITU-R Recommendation P.834-3. "Effects of tropospheric refraction on radiowave propagation". International Telecommunications Union, Geneva, October. 1999.

[8] Vasseur H. "Prediction of Tropospheric Scintillation on Satellite Link from Radiosonde Data". IEEE Transaction on Antenna Propagation. 1999; 47(2): 293-301.

[9] Ortgies G. "Prediction of Slant-Path Amplitude Scintillations from Meteorological Parameters". International Symposium on Radio Propagation, China. 1993.

[10] Otung IE. "Prediction of Tropospheric Amplitude Scintillation on a satellite Link". IEEE Transaction Antenna Propagation. 1996; 44(12): 1600-1608.

[11] Mayer CE. "Evaluation of 8 Scintillation Models". Proceedings of the URSI General Assembly. 2002. 